\title{
Influence of Gas-Surface Interaction on Hypersonic Aerothermodynamic Performance of Flat-Nose Power-Law Bodies
}

\author{
Wilson F. N. Santos* \\ National Institute for Space Research \\ Cachoeira Paulista, SP 12630-000 BRAZIL
}

The present work performs a computational study on rarefied hypersonic flow past flatnose leading edges at zero incidence. Effects of incomplete surface accommodation on the aerothermodynamic surface quantities have been investigated by employing the Direct Simulation Monte Carlo (DSMC) method in combination with the Cercignani-Lampis-Lord gas-surface interaction model, which incorporates separate accommodation coefficients for normal and tangential velocity components. The work also focuses the attention of designers of hypersonic configurations on the fundamental parameter of bluntness, which can have an important impact on even initial design. The results presented highlight the sensitivity of the heat transfer and drag coefficients to changes on the gas-surface accommodation coefficients. These are compared to the results for classical diffuse reflection model. It was found that stagnation point heating decreased by a reduction on the normal accommodation coefficient and the total drag decreased by a reduction on the tangential accommodation coefficient.

\section{Nomenclature}

a Constant in power-law body equation, Eq.(2)

$C_{d} \quad$ Drag coefficient, $2 D / \rho_{\infty} V_{\infty}^{2} H$

$C_{f} \quad$ Skin friction coefficient, $2 \tau_{w} / \rho_{\infty} V_{\infty}^{2}$

$C_{h} \quad$ Heat transfer coefficient, $2 q_{w} / \rho_{\infty} V_{\infty}^{3}$

$C_{p} \quad$ Pressure coefficient, $2\left(p_{w}-p_{\infty}\right) / \rho_{\infty} V_{\infty}^{2}$

$D$ Drag force, $\mathrm{N}$

d Molecular diameter, $\mathrm{m}$

$H \quad$ Body height at the base, $\mathrm{m}$

$K n$ Knudsen number, $\lambda / l$

$L \quad$ Body length, m

$l \quad$ Characteristic length, $\mathrm{m}$

$M \quad$ Mach number

$m \quad$ Molecular mass, $\mathrm{kg}$

$N \quad$ Number flux, $\mathrm{m}^{-2} s^{-1}$

$n \quad$ Body power law exponent

$p$ Pressure, $\mathrm{N} / \mathrm{m}^{2}$

$q \quad$ Heat flux, $\mathrm{W} / \mathrm{m}^{2}$

${ }^{*}$ Researcher, Combustion and Propulsion Laboratory. AIAA Member. 


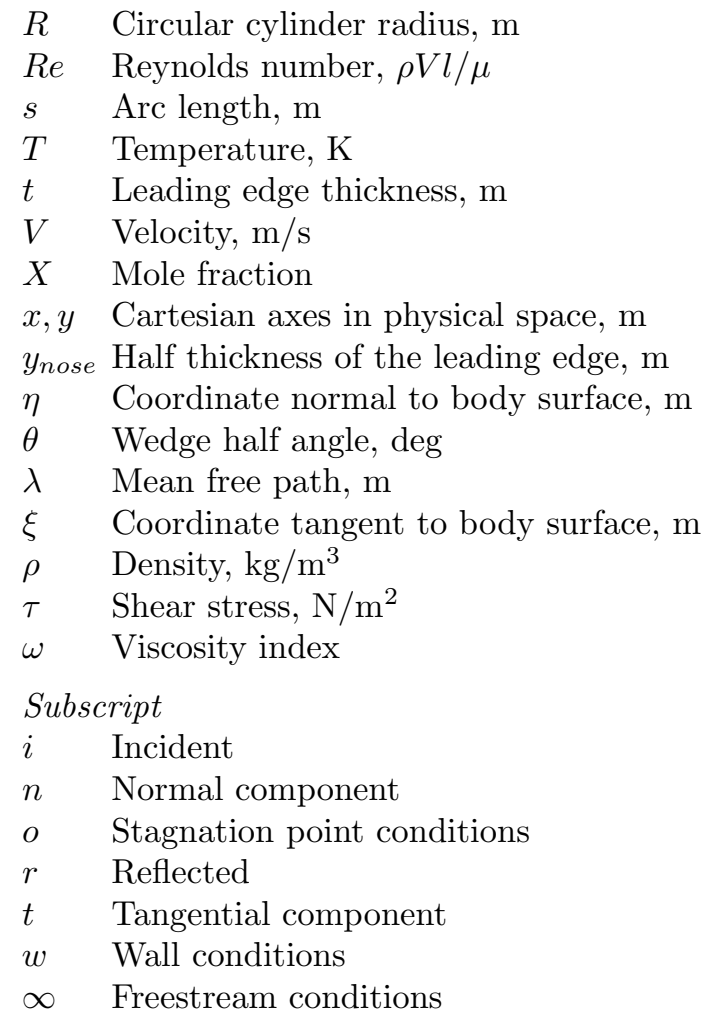

\section{Introduction}

7 HE problems related to the aerothermodynamics at high flight Mach numbers have recently received the

1 attention of several investigations because of their importance in connection with hypersonic vehicles and re-entry problems. Hypersonic vehicles are generally characterized by slender bodies and sharp leading edges in order to achieve good aerodynamic properties like high lift and low drag. Nevertheless, at high Mach numbers, the vehicle leading edges should be sufficiently blunt in order to reduce the heat transfer rate to acceptable levels, and possibly to allow for internal heat conduction. In addition, as aerodynamic heating may cause serious problems at these speeds, the removal of heat near the front of the leading edge must be considered, since the stagnation region is one of the most thermally stressed zones. Therefore, designing a hypersonic vehicle leading edge involves a tradeoff between making the leading edge sharp enough to obtain acceptable aerodynamic and propulsion efficiency and blunt enough to reduce the aerodynamic heating at the stagnation point.

Recently, considerable attention has been given to the problem of calculating aerodynamic characteristics of power law bodies $\left(y \propto x^{n}, 0<n<1\right)$ at hypersonic speed ${ }^{1-11}$. The major interest in these works has gone into considering the power-law shapes as possible candidates for blunting geometries of hypersonic leading edges, such as hypersonic waverider vehicles ${ }^{12}$ which have been lately considered for high-altitude/low-density applications ${ }^{13-17}$. The interest in power law shapes is based on the work of Mason and Lee ${ }^{18}$, who have pointed out that, for certain exponents, power law shapes exhibit aerodynamic properties similar to geometrically sharp shapes. They suggested the possibility of a difference between shapes that are geometrically sharp and shapes that behave aerodynamically as if they were sharp.

Of particular significance on power law shapes are the works by Santos and Lewis ${ }^{3-10}$. For the idealized situation of two-dimensional rarefied hypersonic flow, they found that the stagnation point heating behavior for power law leading edges with finite radius of curvature $(n=1 / 2)$ followed that predicted for classical 
blunt body, i.e., the heating rate on blunt bodies is inversely proportional to the square root of curvature radius at the stagnation point. For those power law leading edges with zero radii of curvature $(n>1 / 2)$, it was found that the stagnation point heating is not a function of the curvature radius at the vicinity of the leading edges, but agreed with the classical blunt body behavior predicted by the continuum flow far from the stagnation point. Results were compared to a corresponding circular cylinder to determine which geometry would be better suited as a blunting profile. Their analysis also showed that power law shapes provided smaller total drag and smaller shock wave standoff distance than the circular cylinder, typically used in blunting sharp leading edges for heat transfer considerations. However, circular cylinder provided smaller stagnation point heating than the power law shapes under the range of conditions investigated.

In order to improve the stagnation point heating of power law shapes, a modification was introduced into the power law leading edges. The proposed leading edge is composed of a flat nose followed by an afterbody surface defined by a power law shape, the flat-nose power-law leading edge. This concept is based on the work of Reller ${ }^{19}$, who showed that a method of designing low heat transfer bodies is devised on the premise that the rate of heat transfer to the nose will be low if the local velocity is low, while the rate of heat transfer to the afterbody will be low if the local density is low. According to Reller ${ }^{19}$, a typical body that results from this design method consists of a flat nose followed by a highly curved, but for the most part slightly inclined, afterbody surface.

In this context, Santos ${ }^{20-22}$ has examined the aerodynamic surface quantities for a family of these new contours, flat-nose power-law leading edges. The emphasis of the works was to compare the performance of these new contours with that for power-law leading edges with zero-thickness nose (Santos and Lewis ${ }^{3}$ ). The thickness effect was examined for a range of Knudsen number, $K n_{t}$, based on the leading edge thickness, covering from the transitional flow regime to the free molecular flow one. It was examined a group of shapes that combined $K n_{t}$ of 1, 10 and 100 and power law exponents of 2/3, 3/4 and 4/5. The analysis showed that flat-nose power-law leading edges provided much smaller stagnation point heating and slightly larger total drag than the power law shapes (zero-thickness nose) under the range of conditions investigated.

These works ${ }^{20-22}$ on hypersonic flow past flat-nose power-law shapes have been concentrated primarily on the analysis of the aerothermodynamic surface quantities by considering the diffuse reflection model as being the gas-surface interaction. The diffuse model assumes that the molecules are reflected equally in all directions, quite independently of their incident speed and direction. Nonetheless, as a space flight vehicle is exposed to a rarefied environment over a considerable time, a departure from the fully diffuse model is observed, resulting from the colliding molecules that clean the surface of the vehicle, which becomes gradually decontaminated. Molecules reflected from clean surfaces show lobular distribution in direction ${ }^{23}$. The flux distribution of scattered molecules emitted from clean surfaces frequently has a lobular shape that is centered about an angle which tends to approach the specular angle for very high energies and/or low angle of attack.

Both the aerodynamic surface quantities and the state of the gas adjacent to the body surface are very sensitive to the assumptions used in the calculation concerning the gas-surface interaction model for transitional and free molecular flows. In addition, the essential phenomena of rarefied gases are found mostly in the region relatively near to the solid boundaries, i.e., within a few mean free paths. Thus a knowledge of the physics of the interaction of gas molecules and solid surfaces is of primary importance.

In an effort to obtain further insight into the nature of the flowfield structure of flat-nose power-law leading edges under hypersonic transitional flow conditions, a study is performed on these shapes with a great deal of emphasis placed on the gas-surface interaction effects. In this scenario, the primary goal of this paper is to assess the sensitivity of the aerodynamic surface quantities to variations on the surface accommodation coefficients experienced by the leading edges by employing the Cercignani-Lampis-Lord (CLL) model ${ }^{24}$. The CLL model is implemented into the DSMC code, and simulations are performed by assuming two-dimensional rarefied hypersonic flow. 


\section{Gas-Surface Interaction Model}

The successful application of the DSMC method requires the development of accurate gas-surface interaction model besides the gas-gas molecular collision model. As the majority of the practical problems involves gas-surface interaction phenomena, a suitable boundary condition is required in order to obtain reliable results from numerical simulation of rarefied gas flows.

Three models of gas-surface interactions may be employed in the DSMC method as practical models for purpose of engineering surfaces: (1) specular reflection, (2) diffuse reflection, and (3) some combination of these. In a specular reflection, molecules are reflected like a perfectly elastic sphere with reversal of the normal component of velocity and no change in the parallel component of velocities and energy. In a diffuse reflection, the molecules are reflected equally in all directions usually with a complete thermal accommodation. The final velocity of the molecules is randomly assigned according to a half-range Maxwellian distribution determined by the wall temperature. The combination of diffuse reflection with specular reflection (Maxwell model) introduces a single parameter $f$ to indicate the fraction of those molecules reflected diffusely in a completely accommodated fashion according to a Maxwellian distribution corresponding to the wall temperature, and the remaining fraction (1- $f$ ), being assumed to reflect specularly.

The Maxwell model was followed by the introduction of three accommodation coefficients that describe the degree of accommodation of the incident normal momentum, tangential momentum and kinetic energy to those of the surface. The traditional definition ${ }^{25}$ for these coefficients are usually expressed as being,

$$
\sigma_{n}=\frac{p_{i}-p_{r}}{p_{i}-p_{w}}, \quad \sigma_{t}=\frac{\tau_{i}-\tau_{r}}{\tau_{i}}, \quad \alpha=\frac{e_{i}-e_{r}}{e_{i}-e_{w}}
$$

where terms $p, \tau$ and $e$ refer to the momentum flux acting normal and tangential to the surface, and the energy flux to the surface per unit area per unit time, respectively; subscripts $i$ and $r$ stand for the incident and reflected components, and $w$ refers to the component that would be produced by a diffuse reflection at the temperature of the surface.

Data from many experiments show that molecules reflected or re-emitted from solid surfaces present lobular distributions under high vacuum conditions and are poorly represented by the Maxwell model. However, this model is widely used because it satisfies the principle of detailed balance or reciprocity. Detailed balance means that at equilibrium every molecular process and its inverse process must individually balance.

A phenomenological model that satisfies detailed balance and has demonstrated improvement over the Maxwell model has been proposed by Cercignani and Lampis ${ }^{26}$ (CL model). This model is based on the definition of the accommodation coefficients $\alpha_{n}$ and $\alpha_{t}$ that represent the accommodation coefficients for the kinetic energy associated with the normal and tangential components of velocity. The CL model provides a continuous spectrum of behavior from specular reflection at one end to diffuse reflection with complete energy accommodation at the other, and produces physically realistic distributions of direction and energy re-emitted molecules. Lord ${ }^{24}$ has shown that the CL model is suited for the DSMC method ${ }^{27}$, and described how to incorporate it into the DSMC method. The DSMC method with Lord's implementation is referred as the Cercignani-Lampis-Lord (CLL) method. The CLL model is derived assuming that there is no coupling between the normal and tangential momentum components. The two adjustable parameters appearing in the CLL model are the normal component of translational energy $\alpha_{n}$ and the tangential component of momentum $\sigma_{t}$. Figure 1 displays a schematic comparison of the Maxwell reflection model and the CLL reflection model.

In order to simulate the partial surface accommodation, the Cercignani-Lampis-Lord (CLL) model ${ }^{24}$ was implemented in this DSMC calculation. However, in the implementation process, Bird ${ }^{27}$ has shown that it is equivalent to specify the normal $\alpha_{n}$ and tangential $\alpha_{t}$ components of translational energy, since $\alpha_{t}=\sigma_{t}\left(2-\sigma_{t}\right)$, and thus that $\sigma_{t}<\alpha_{t}$, assuming that $\sigma_{t}$ lies between 0 and 1 . In the present simulations, $\alpha_{n}$ and $\sigma_{t}$ are used as being the two adjustable parameters. It is important to mention that in the CLL model the accommodation of internal energy is allowed to be independent of the translational accommodation.

4 of 16 


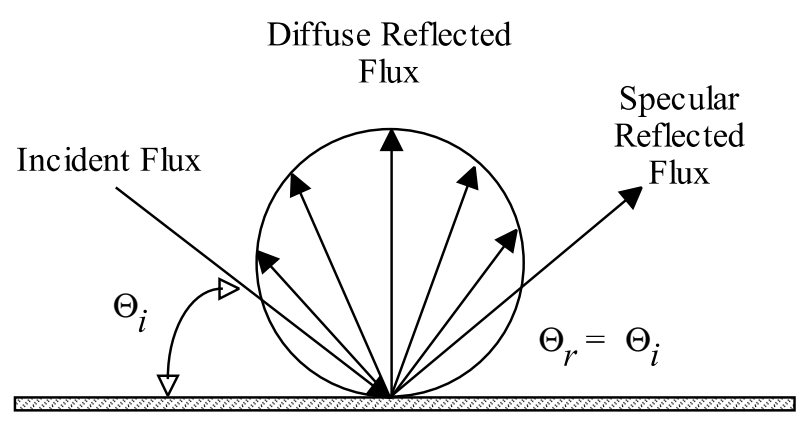

(a) Maxwell model

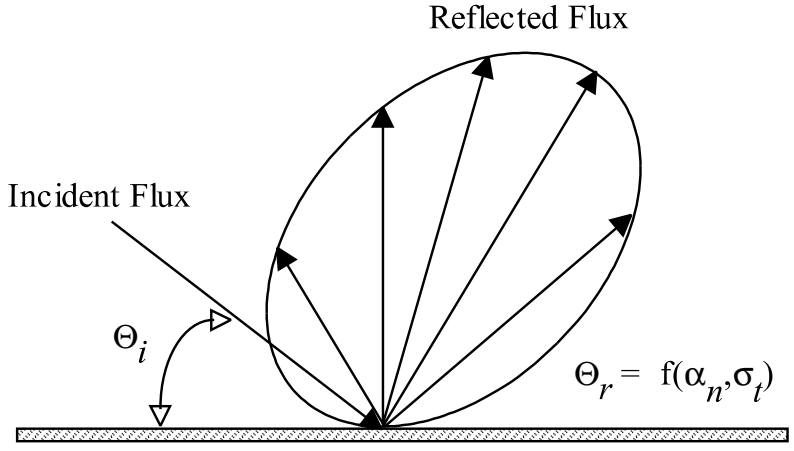

(b) CLL model

Figure 1. Drawing illustrating the (a) Maxwell and (b) the CLL reflection model.

\section{Leading Edge Geometry Definition}

In dimensional form, the power law contours that define the shapes of the afterbody surfaces are given by the following expression,

$$
y=y_{\text {nose }}+a x^{n}
$$

where $y_{n o s e}$ is the half thickness of the flat nose of the leading edges, $n$ is the power law exponent and $a$ is the power law constant which is a function of $n$.

The flat-nose power-law shapes are modeled by assuming a sharp leading edge (wedge) of half angle $\theta$ with a circular cylinder of radius $R$ inscribed tangent to this wedge. The flat-nose power law shapes, inscribed between the wedge and the cylinder, are also tangent to both shapes at the same common point where they have the same slope angle. It was assumed a leading edge half angle of 10 degree, a circular cylinder diameter of $10^{-2} \mathrm{~m}$, power law exponents of $2 / 3,3 / 4$, and $4 / 5$, and front surface thicknesses $t / \lambda_{\infty}$ of $0.01,0.1$ and 1 , where $t=2 y_{\text {nose }}$ and $\lambda_{\infty}$ is the freestream molecular mean free path. Figure 2 shows schematically this construction.

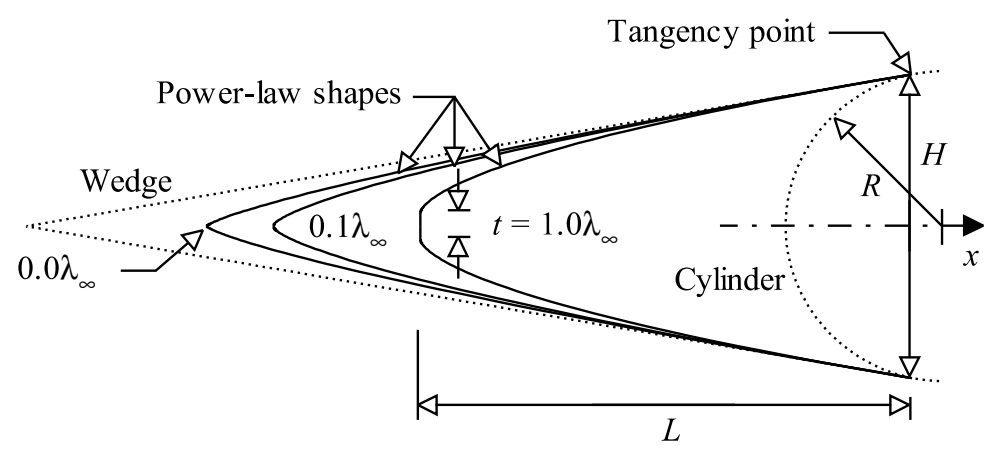

Figure 2. Drawing illustrating the leading edge geometries.

From geometric considerations, the power law constant $a$ is obtained by matching slopes for the wedge, circular cylinder and power law body at the tangency point. The common body height $H$ at the tangency 
point is equal to $2 R \cos \theta$, and the body length $L$ from the nose to the tangency point in the axis of symmetry is given by $n(H-t) / 2 \tan \theta$. It was assumed that the flat-nose power-law bodies are infinitely long but only the length $L$ is considered, since the wake region behind the bodies is not of interest in this investigation.

\section{Computational Method and Procedure}

The DSMC method ${ }^{27}$ has become today the most valuable technique for the investigation of rarefied gases. The DSMC method does not solve a system of equations to produce a solution of the flowfield, but rather statistically tracks movements and collisions of simulated molecules, each of which represents a fixed number of real gas molecules. In the movement phase, all particles are moved over distances appropriate to a short time interval, time step, and some of them interact with the domain boundaries in this time interval. Particles that strike the solid wall would reflect according to the appropriate gas-surface interaction model. In the collision phase, intermolecular collisions are performed according to the theory of probability without time being consumed. In this context, the intermolecular collisions are uncoupled to the translational molecular motion over the time step used to advance the simulation. Time is advanced in discrete steps such that each step is small in comparison with the mean collision time ${ }^{27}$. The method does not require an initial approximation to the flowfield and there is no iterative procedure for convergence to the final solution.

The physical models employed in the present simulations are as follows. Intermolecular collisions are treated by using the variable hard sphere (VHS) molecular model ${ }^{28}$ and the no time counter (NTC) collision sampling technique ${ }^{29}$. Energy partitioning is accounted for using the Borgnakke-Larsen statistical model ${ }^{30}$ with constant rotational and vibrational collision numbers, 5 for rotation and 50 for vibration. Simulations are performed using a non-reacting gas model consisting of two chemical species, $\mathrm{N}_{2}$ and $\mathrm{O}_{2}$.

In order to implement the particle-particle collisions, the flowfield is divided into an arbitrary number of regions, which are subdivided into computational cells. The cells are further subdivided into four subcells, two subcells/cell in each direction. The cell provides a convenient reference sampling of the macroscopic gas properties, whereas the collision partners are selected from the same subcell for the establishment of the collision rate.

The computational domain used for the calculation is made large enough so that body disturbances do not reach the upstream and side boundaries, where freestream conditions are specified. A schematic view of the computational domain is depicted in Fig. 3. Side I is defined by the body surface. Reflection with incomplete surface accommo-

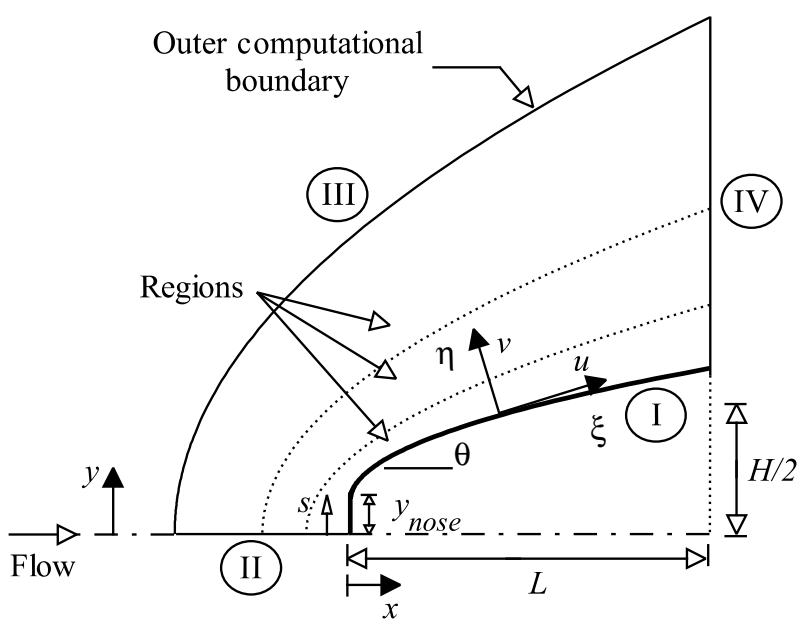
dation is the condition applied to this side. Advantage of the flow symmetry is taken into account, and molecular simulation is applied to one-half of a full configuration. Therefore, side II is a plane of symmetry. In such a boundary, all flow gradients normal to the plane are zero. At the molecular level, this plane is equivalent to a specular reflecting boundary. Side III is the freestream side through which simulated molecules enter and exit. Finally, the flow at the downstream outflow boundary, side IV, is predominantly supersonic and vacuum condition is specified ${ }^{27}$. At this boundary, simulated molecules can only exit.

Numerical accuracy in DSMC method depends on the grid resolution chosen as well as the number of particles per computational cell. The effect of grid resolution on computed results is of particular interest for the present study because insufficient grid resolution can reduce significantly the accuracy of the predicted aerodynamic heating and forces acting on the body surface. Hence, heat transfer, pressure and skin friction 
coefficients are used as the representative parameters for the grid sensitivity. Grid independence was tested by running the calculations with half and double the number of cells in $\xi$ and $\eta$ directions (see Fig. 3) compared to a standard grid. Solutions (not shown) were near identical for all grids used and were considered fully grid independent.

\section{Flow Conditions}

The freestream flow conditions used for the numerical simulation of flow past the leading edges are those given by Santos $^{20}$ and summarized in Table 1, and the gas properties ${ }^{27}$ are shown in Table 2.

Table 1. Freestream flow conditions

\begin{tabular}{ccccccc}
\hline \hline Altitude $(\mathrm{km})$ & $T_{\infty}(\mathrm{K})$ & $p_{\infty}\left(\mathrm{N} / \mathrm{m}^{2}\right)$ & $\rho_{\infty}\left(\mathrm{kg} / \mathrm{m}^{3}\right)$ & $\mu_{\infty}\left(\mathrm{Ns} / \mathrm{m}^{2}\right)$ & $n_{\infty}\left(\mathrm{m}^{-3}\right)$ & $\lambda_{\infty}(\mathrm{m})$ \\
\hline 70 & 220.0 & 5.582 & $8.753 \times 10^{-5}$ & $1.455 \times 10^{-5}$ & $1.8209 \times 10^{21}$ & $9.03 \times 10^{-4}$ \\
\hline \hline
\end{tabular}

The freestream velocity $V_{\infty}$ is assumed to be constant at $3.5 \mathrm{~km} / \mathrm{s}$, which corresponds to a freestream Mach number $M_{\infty}$ of 12 . The translational and vibrational temperatures in the freestream are in equilibrium at $220 \mathrm{~K}$, and the leading edge surface has a constant wall temperature $T_{w}$ of $880 \mathrm{~K}$ for all cases considered. The freestream Reynolds number by unit meter $R e_{\infty}$ is 21455 based on conditions in the undisturbed stream.

The overall Knudsen number $K n_{t}$, defined as $\lambda_{\infty} / t$, corresponds to 100,10 and 1 for thickness, $t / \lambda_{\infty}$ of $0.01,0.1$ and 1 , respectively. It is important to mention that $t / \lambda_{\infty}=0$ case $\left(K n_{t}=\infty\right)$ corresponds to the power law leading edge set already investigated by Santos and Lewis ${ }^{3}$.

In order to simulate the incomplete surface accommodation, $\alpha_{n}$ and $\sigma_{t}$ are used as being the two adjustable parameter. The DSMC calculations were performed independently for three distinct numerical values for $\alpha_{n}$ and $\sigma_{t}: 0.5,0.75$ and 1. $\alpha_{n}$ and $\sigma_{t}$ equal to 1 represents the diffusion reflection. In addition, the internal energy accommodation was kept equal to one for all calculations presented in this work.

\section{Computational Results and Discussion}

Aerodynamic surface quantities of particular interest are number flux, heat transfer, wall pressure, skin friction and drag. Therefore, the purpose of this section is to discuss and to compare differences in the profiles of these properties, expressed in coefficient form, due to variations on the normal and tangential accommodation coefficients associated to the gas-surface interaction.

\section{A. Number Flux}

The number flux $N$ is calculated by sampling the molecules impinging on the surface by unit time and unit area. The dependence of the number flux on the normal $\alpha_{n}$ and tangential $\sigma_{t}$ accommodation coefficients is shown in Fig. 4 for a group of flat-nose power-law bodies. Figures $4(\mathrm{a})$ and (b) represent sharp leading edges defined by nose thickness corresponding to $K n_{t}=100\left(t / \lambda_{\infty}=0.01\right)$ with afterbody defined by $n=$ $2 / 3$ and $4 / 5$, respectively. Figures $4(\mathrm{c})$ and (d) represent the blunt leading edges defined by nose thickness corresponding to $K n_{t}=1\left(t / \lambda_{\infty}=1\right)$ with afterbody also defined by $n=2 / 3$ and $4 / 5$, respectively. The 

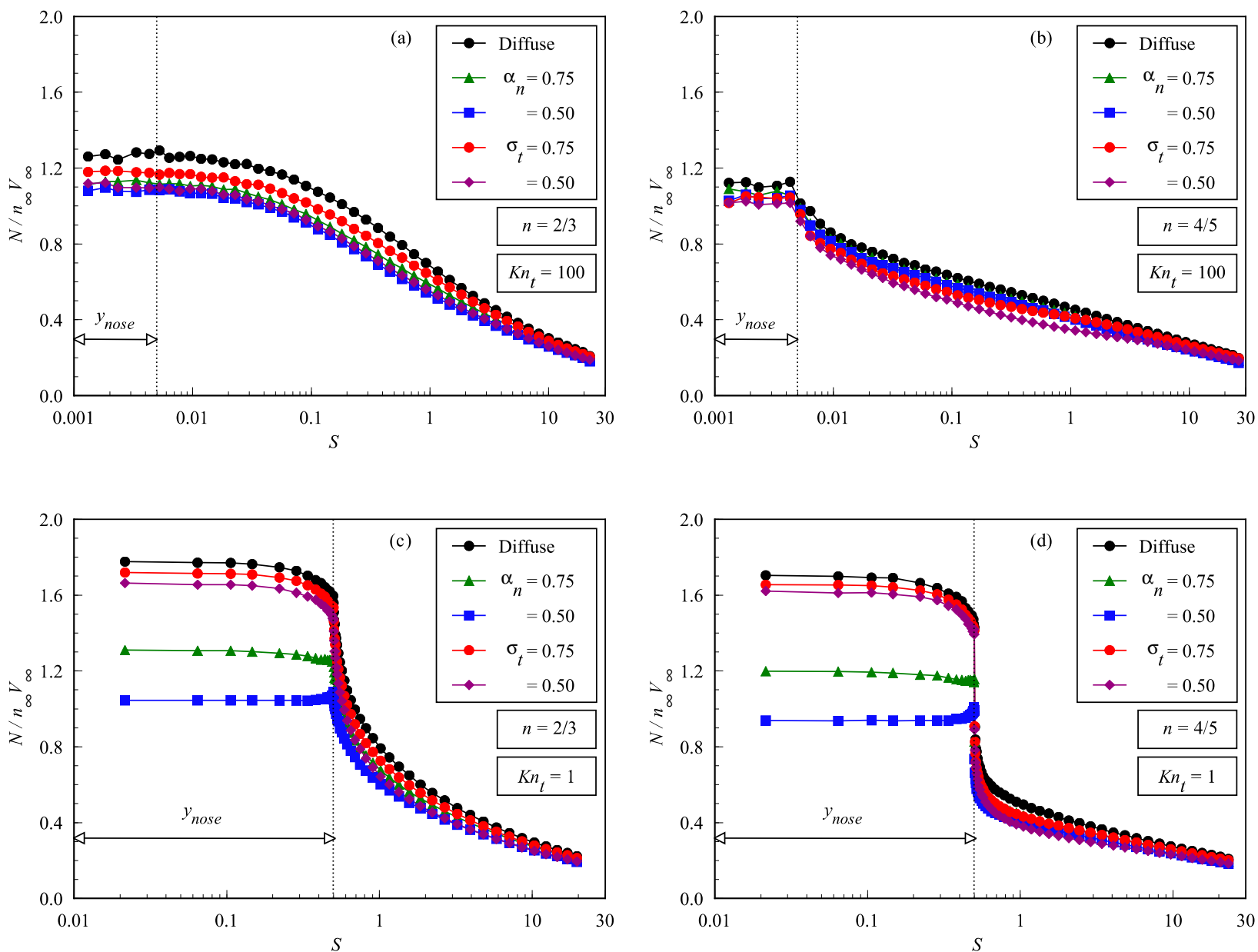

Figure 4. Effect of normal $\alpha_{n}$ and tangential $\sigma_{t}$ accommodation coefficients on number flux $N / n_{\infty} V_{\infty}$ along the body surface for (a) $K n_{t}=100$ and $n=2 / 3$, (b) $K n_{t}=100$ and $n=4 / 5$, (c) $K n_{t}=1$ and $n=2 / 3$, and (d) $K n_{t}=1$ and $n=4 / 5$.

other cases involving a combination of $K n_{t}=10\left(t / \lambda_{\infty}=0.1\right)$ and $n=3 / 4$ are intermediate to the cases illustrated in Fig. 4, and they will not be shown. In this set of plots, the number flux $N$ is normalized by $n_{\infty} V_{\infty}$, where $n_{\infty}$ stands for the freestream number density and $V_{\infty}$ for the freestream velocity, and $S$ is the arc length $s$ normalized by the freestream mean free path $\lambda_{\infty}$.

According to this set of plots, it is clearly seen that the dimensionless number flux to the surface depends not only on the gas-surface interaction but also on the combination of leading edge thickness and afterbody shape. For sharp leading edge, $K n_{t}=100$ and $n=2 / 3$, Fig. $4(\mathrm{a})$, the dimensionless number flux is low and constant along the frontal surface and decreases gradually along the afterbody surface. As the afterbody shape changes from $n=2 / 3$ to $4 / 5$, the number flux stays constant along the frontal surface, but decreases sharply in the vicinity of the flat-face/afterbody junction. For blunt leading edge, $K n_{t}=1$ and $n=2 / 3$, Fig. 4(c), the dimensionless number flux is large on the frontal surface. It presents a constant value in the first half of the frontal surface and decreases in the vicinity of the shoulder. After that, it decreases significantly along the afterbody surface. This increase in the dimensionless number flux with increasing the leading edge thickness may be related to the collisions of two groups of molecules; the molecules reflecting from the body 
and the molecules oncoming from the freestream. The molecules that are reflected from the body surface, which have a lower kinetic energy interact with the oncoming freestream molecules, which have a higher kinetic energy. Thus, the surface-reflected molecules collide again with the body surface, which produce an increase in the dimensionless number flux in this region. As expected, this behavior is less pronounced by a reduction on the normal or on the tangential accommodation coefficients, since the molecules are reflected from the surface with different energies. Consequently, the net buildup of particle density near the body surface is reduced.

The number flux along the front surface is very sensitive to changes in the normal accommodation coefficient. It is seen that the number flux dramatically decreases with decreasing the normal accommodation coefficient. In contrast, it slightly decreases by a reduction in the tangential accommodation coefficient.

\section{B. Heat Transfer Coefficient}

The heat flux $q_{w}$ to the body surface is calculated by the net energy flux of the molecules impinging on the surface. The net heat flux $q_{w}$ is related to the sum of the translational, rotational and vibrational energies of both incident and reflected molecules. A flux is regarded as positive if it is directed toward the body surface. The heat flux is normalized by $\rho_{\infty} V_{\infty}^{3} / 2$ and presented in terms of heat transfer coefficient $C_{h}$.

The effect of changing the normal $\alpha_{n}$ and the tangential $\sigma_{t}$ accommodation coefficients on the heat transfer coefficient $C_{h}$ is plotted in Fig. 5 as a function of the dimensionless arc length $S$. According to these diagrams, the heat transfer coefficient remains essentially constant over the front surface for sharp (Figs. 5(a) and (b)) and blunt (Figs. 5(c) and (d)) leading edges. Subsequently, $C_{h}$ increases in the vicinity of the flatface/afterbody junction for the blunt cases investigated, $K n_{t}=1\left(t / \lambda_{\infty}=1\right)$. The heat transfer coefficient $C_{h}$ decreases sharply at the vicinity of the flat-face/afterbody surface and continues to decline along the body surface. Similar to the number flux, the net heat transfer coefficient decreases with decreasing $\alpha_{n}$ for sharp and blunt leading edges. Nevertheless, the heat transfer coefficient increases slightly by a reduction on $\sigma_{t}$ provided the leading edge is sharp.

Usually, the stagnation region is considered as being one of the most thermally stressed zones in either sharp or blunt bodies, as shown by the power-law cases, defined by $t / \lambda_{\infty}=0$, investigated by santos and Lewis $^{3}$. Nonetheless, as a flat nose is introduced in these power-law shapes, the most severe heat transfer region moves to the flat-face/afterbody junction. As the number of molecules impinging on the front surface decreases in the vicinity of the flat-face/afterbody junction (see Fig. 4), then the velocity of the molecules increases as the flow approaches the junction of the leading edge in order to increase the heat transfer coefficient. Moreover, the contribution of the translational energy to the net heat flux varies with the square of the molecular velocity.

At this point, it seems important to compare the heat transfer coefficient at the stagnation point $C_{h o}$ of the flat-nose power-law shapes $\left(C_{h o}\right)$ for diffusion reflection with that by considering reflection with partial accommodation coefficient. Table 3 displays the ratio $C_{h o} / C_{h o, d i f f}$ for the cases investigated as a function of the Knudsen number $K n_{t}$ and power law exponent $n$. According to Table 3, a substantial reduction in the heat transfer coefficient at the stagnation point is obtained by reducing the normal accommodation coefficient $\alpha_{n}$. Also, it is apparent from Table 3 that the leading edges with nose thickness of $K n_{t}=10$ present the lowest values for heat transfer at the stagnation point.

\section{Pressure Coefficient}

The pressure $p_{w}$ on the body surface is calculated by the sum of the normal momentum fluxes of both incident and reflected molecules at each time step. Results are presented in terms of the pressure coefficient $C_{p}$ defined as being $2\left(p_{w}-p_{\infty}\right) / \rho_{\infty} V_{\infty}^{2}$

The variation of the pressure coefficient $C_{p}$ caused by changes on the normal $\alpha_{n}$ and on the tangential $\sigma_{t}$ accommodation coefficients is demonstrated in Fig. 6. It can be noted from these figures that the pressure coefficient is high along the front surface, basically a constant value along it, and decreases dramatically along 

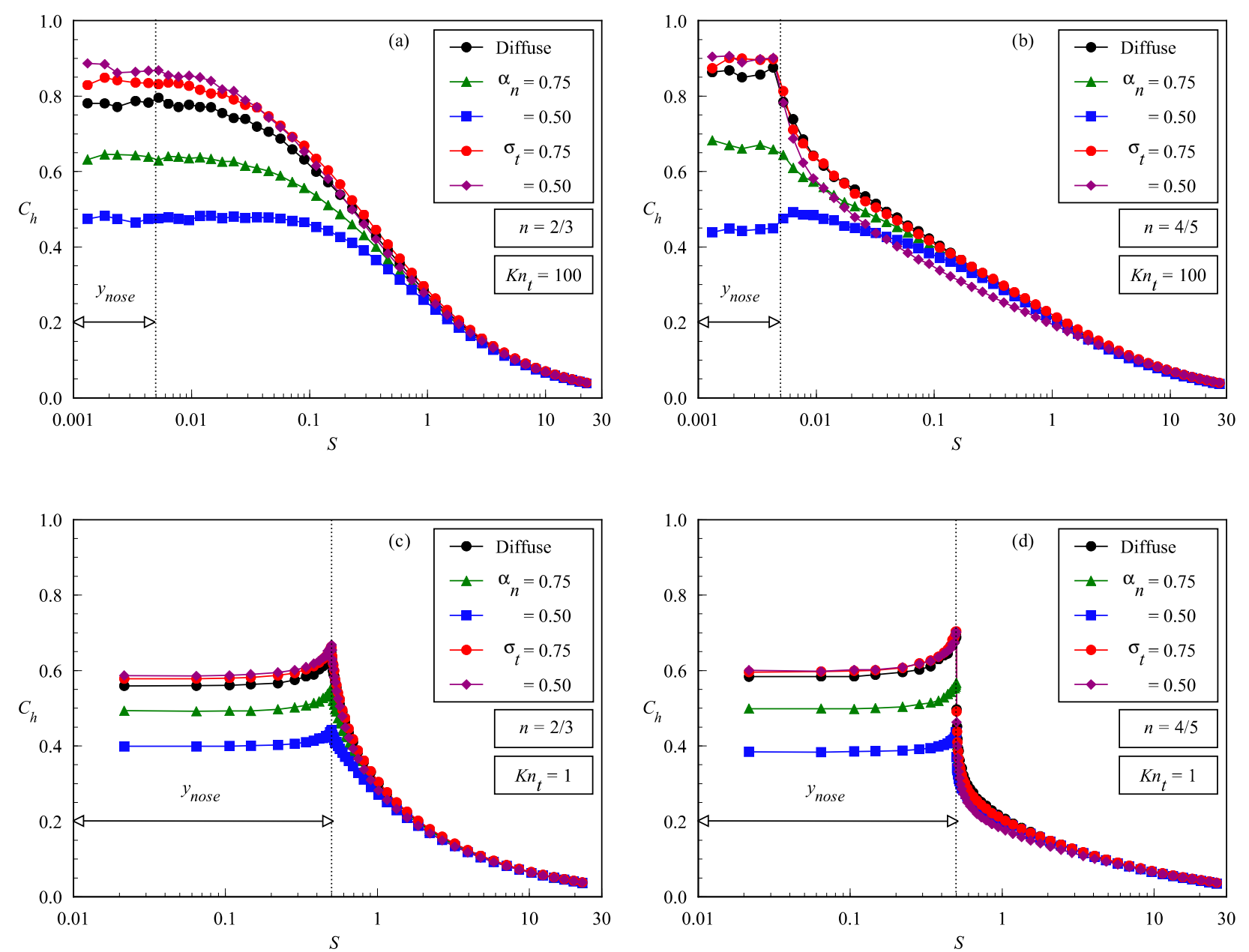

Figure 5. Effect of (a) normal $\alpha_{n}$ and (b) tangential $\sigma_{t}$ accommodation coefficients on heat transfer coefficient $C_{h}$ along the body surface for (a) $K n_{t}=100$ and $n=2 / 3$, (b) $K n_{t}=100$ and $n=4 / 5$, (c) $K n_{t}=1$ and $n=$ $2 / 3$, and (d) $K n_{t}=1$ and $n=4 / 5$.

Table 3. Heat transfer coefficient comparison at the stagnation point, $C_{h o} / C_{h o, d i f f}$.

\begin{tabular}{cccccc}
\hline \hline Exp. $n$ & $\alpha_{n}$ & $\sigma_{t}$ & $K n_{t}=1$ & $K n_{t}=10$ & $K n_{t}=100$ \\
\hline $2 / 3$ & $0.75(0.50)$ & 1 & $0.880(0.712)$ & $0.799(0.592)$ & $0.823(0.612)$ \\
$2 / 3$ & 1 & $0.75(0.50)$ & $1.034(1.048)$ & $1.056(1.076)$ & $1.078(1.123)$ \\
$3 / 4$ & $0.75(0.50)$ & 1 & $0.861(0.672)$ & $0.757(0.519)$ & $0.789(0.554)$ \\
$3 / 4$ & 1 & $0.75(0.50)$ & $1.026(1.033)$ & $1.031(1.033)$ & $1.056(1.071)$ \\
$4 / 5$ & $0.75(0.50)$ & 1 & $0.852(0.657)$ & $0.735(0.499)$ & $0.775(0.521)$ \\
$4 / 5$ & 1 & $0.75(0.50)$ & $1.022(1.026)$ & $1.022(1.017)$ & $1.033(1.040)$ \\
\hline \hline
\end{tabular}

10 of 16 

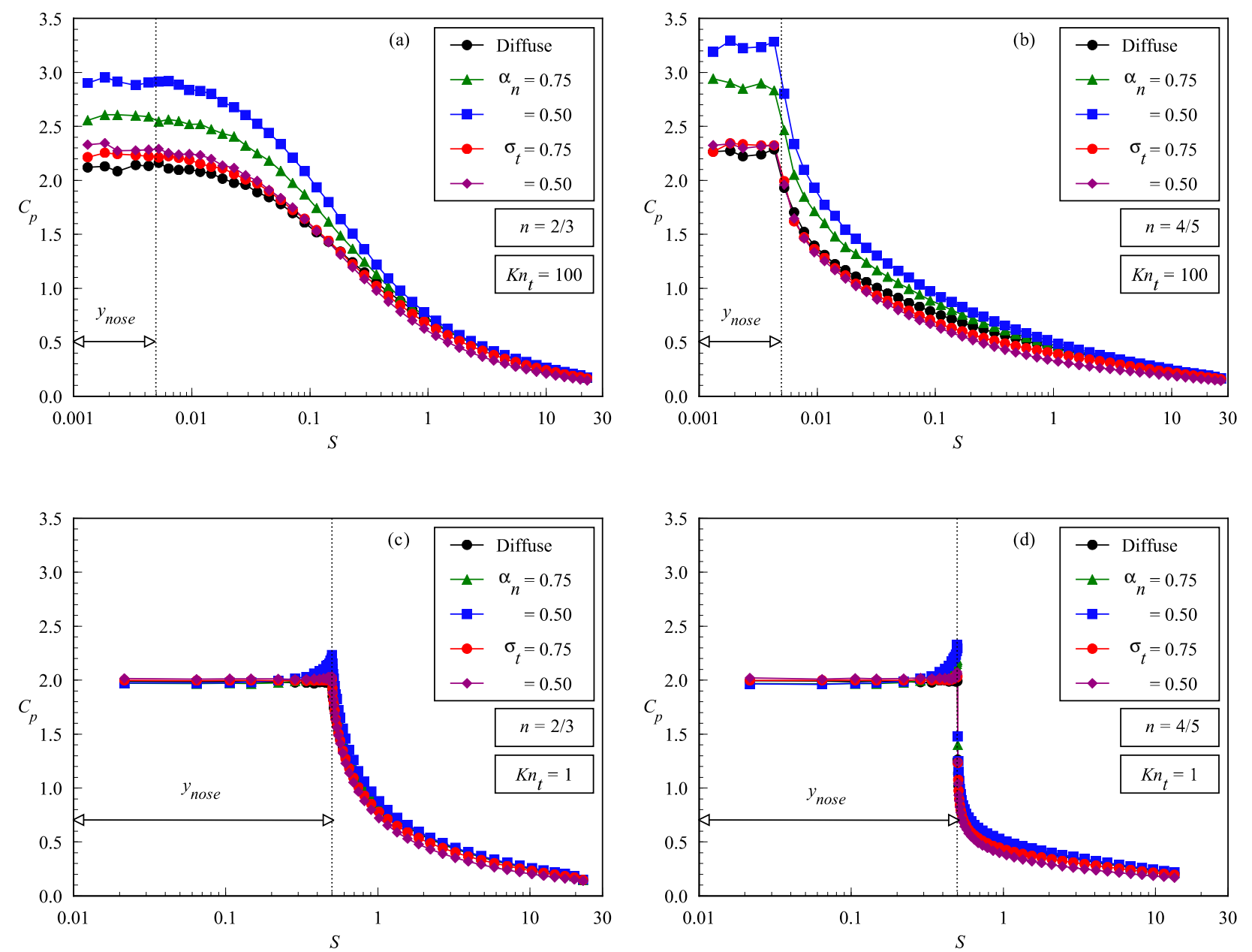

Figure 6. Effect of (a) normal $\alpha_{n}$ and (b) tangential $\sigma_{t}$ accommodation coefficients on pressure coefficient $C_{p}$ along the body surface for (a) $K n_{t}=100$ and $n=2 / 3$, (b) $K n_{t}=100$ and $n=4 / 5$, (c) $K n_{t}=1$ and $n=2 / 3$, and (d) $K n_{t}=1$ and $n=4 / 5$.

the afterbory surface for the combination of thickness and afterbody shape investigated. It is also noticed that the pressure coefficient distributions for the partial accommodation calculations differs from that for full accommodation (diffuse reflection case) along the body surface. For sharp leading edges, Figs. 6(a) and (b), the pressure coefficient increases significantly along the frontal suraface as well as along the afterbody surface. In contrast, for blunt leading edges, Figs. 6(c) and (d), partial accommodation coefficient calculations have no expressive effect on the pressure coefficient, except at the vicinity of the flat-face/afterbody junction. An understanding of this behavior can be gained by analyzing the contributions of the incident and reflected components of the pressure coefficient along the body surface. It may be observed (not shown) that the energetic scattered molecules play a more significant role, since the incident component of the pressure coefficient decreases and the reflected one increases with decreasing the normal and tangential accommodation coefficient. Hence, the insensitivity of the pressure coefficient to accommodation coefficient variations in the range investigated is primarily attributed to a counterbalance between the number flux reduction and the tangential momentum rise related to the reflected molecules. 

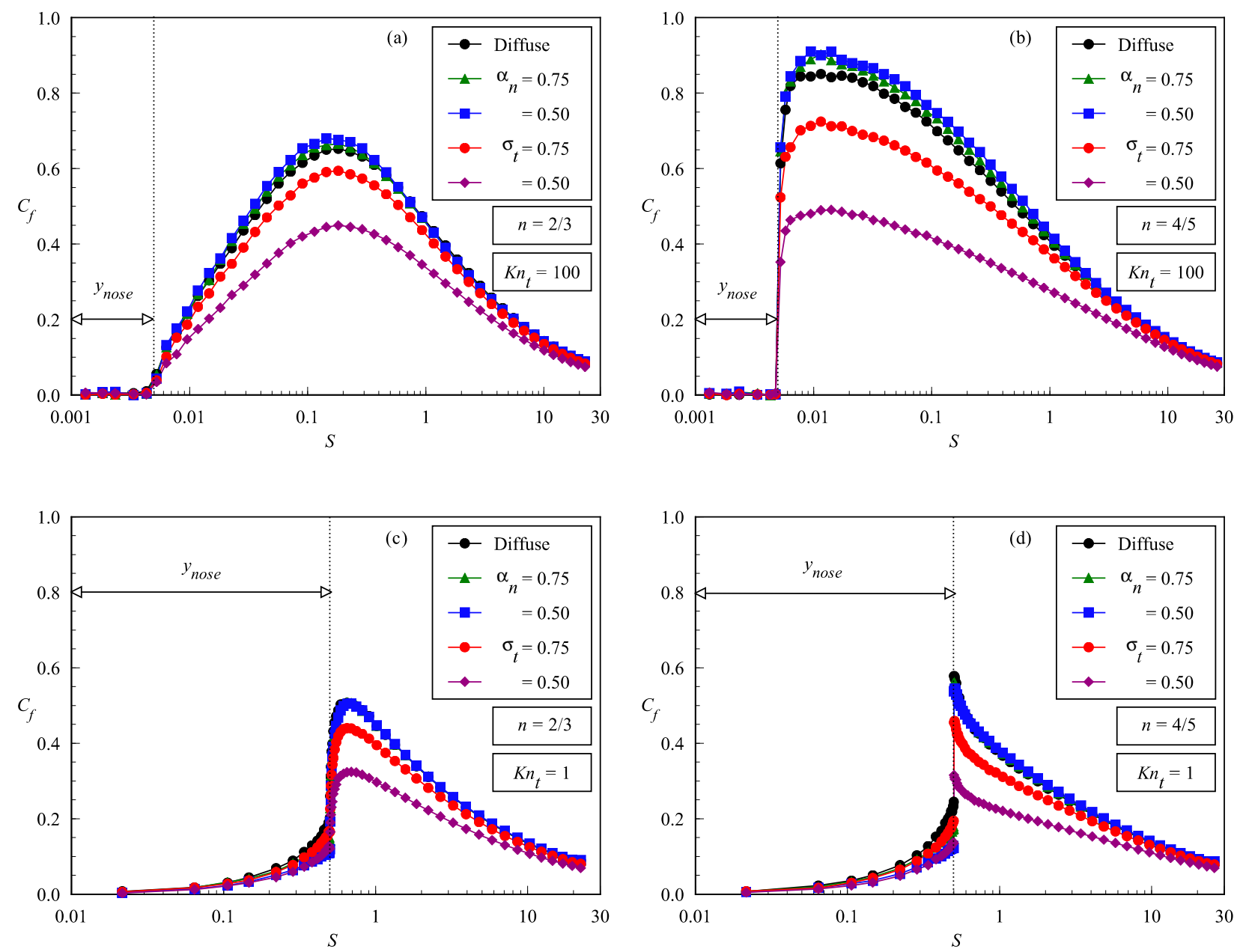

Figure 7. Effect of (a) normal $\alpha_{n}$ and (b) tangential $\sigma_{t}$ accommodation coefficients on skin friction coefficient $C_{p}$ along the body surface for (a) $K n_{t}=100$ and $n=\mathbf{2} / \mathbf{3}$, (b) $K n_{t}=100$ and $n=4 / 5$, (c) $K n_{t}=10$ and $n=$ 2/3, (d) $K n_{t}=10$ and $n=4 / 5$.

\section{Skin Friction Coefficient}

The shear stress $\tau_{w}$ on the body surface is calculated by averaging the tangential momentum transfer of both incident and reflected molecules impinging on the surface at each time step. The shear stress $\tau_{w}$ on the body surface is normalized by $\rho_{\infty} V_{\infty}^{2} / 2$ and presented in terms of the dimensionless skin friction coefficient $C_{f}$.

It is worthwhile to note that for the special case of diffuse reflection, $\alpha_{n}$ and $\sigma_{t}$ equal to 1 , the reflected molecules have a tangential moment equal to zero, since the molecules essentially lose, on average, their tangential velocity components. In this fashion, the contribution of the reflected tangential momentum flux is equal to zero. Nevertheless, for incomplete surface accommodation, the reflected tangential momentum flux contributes to the skin friction coefficient.

The dependence of the skin friction coefficient $C_{f}$ attributed to variations on the normal $\alpha_{n}$ and tangential $\sigma_{t}$ accommodation coefficients is depicted in Fig. 7. Figures 7(a) and (b) display the skin friction coefficient for sharp leading edges with $K n_{t}=100$ and $n=2 / 3$ and $4 / 5$, respectively. In a similar way, Figs. $7(\mathrm{c})$ and 
(d) illustrate for blunt leading edges with $K n_{t}=1$ and $n=2 / 3$ and $4 / 5$, respectively. According to this set of diagrams, the skin friction coefficient $C_{f}$ is zero at the stagnation point and increases along the front surface up to the flat-face/afterbody junction of the leading edge. After that, $C_{f}$ increases meaningfully to a maximum value that depends on the nose thickness and on the afterbody shape, and decreases downstream along the body surface by approaching the skin friction coefficient predicted by the reference case of zero thickness ${ }^{3}$.

It is immediately evident from Fig. 7 that the change in the tangential accommodation coefficient $\sigma_{t}$ from 1 to 0.50 produces substantial differences in the magnitude of the skin friction coefficient, particularly in a region of the body surface that corresponds to a body slope angle around of 45 degree. The direction of change is toward smaller skin friction coefficient as the accommodation coefficient becomes more incomplete. It is apparent that the major influence on the skin friction coefficient comes from the contribution of the reflected momentum flux of the molecules that increases significantly with decreasing $\sigma_{t}$.

\section{E. Total Drag Coefficient}

The drag on a surface in a gas flow results from the interchange of momentum between the surface and the molecules colliding with the surface. The total drag is obtained by the integration of the pressure $p_{w}$ and shear stress $\tau_{w}$ distributions from the stagnation point of the leading edge to the station $L$ that corresponds to the tangent point common to all the leading edges (see Fig. 2). It is important to mention that the values for the total drag were obtained by assuming the shapes acting as leading edges. Therefore, no base pressure effects were taken into account on the calculations. Results for total drag are normalized by $\rho_{\infty} V_{\infty}^{2} H / 2$ and presented as total drag coefficient $C_{d}$ and its components of pressure drag coefficient $C_{p d}$ and the skin friction drag coefficient $C_{f d}$.

The extent of the changes on the total drag coefficient $C_{d}$ with decreasing the normal $\alpha_{n}$ and the tangential $\sigma_{t}$ accommodation coefficients is illustrated in Fig. 8. In a similar way, Figs. 8(a-d) correspond to sharp leading edges, $K n_{t}=100$ and $n=2 / 3$ and $3 / 4$, and blunt leading edges, $K n_{t}=1$ and $n=2 / 3$ and $3 / 4$.

According to this set of plots, it is clearly seen that the total drag coefficient increases slightly by a reduction in the normal accommodation coefficient $\alpha_{n}$, and decreases significantly by a reduction in the tangential accommodation coefficient $\sigma_{t}$. Of particular interest is the contributions of the pressure and skin friction to the total drag. As the leading edge becomes blunter by increasing the frontal surface, $K n_{t}$ increases from 100 to 10, the contribution of the pressure drag to the total drag increases and the contribution of the skin friction drag decreases. In this scenario, the net effect results in a slightly increase in the total drag as the normal accommodation coefficient $\alpha_{n}$ is reduced from 1 to 0.50 . In contrast, the net effect results in a significant decrease in the total drag by a reduction in the tangential accommodation coefficient $\sigma_{t}$. It may also be recognized that a similar behavior is obtained as the afterbody shape $n$ changes from $2 / 3$ to $4 / 5$ by keeping the same frontal surface thickness.

\section{Concluding Remarks}

The computations of a rarefied hypersonic flow on blunt bodies have been performed by using the Direct Simulation Monte Carlo method. The calculations provided information concerning the nature of the aerodynamic surface quantities for a family of contours composed by a flat nose followed by a curved afterbody surface defined by power-law shapes. Effects of incomplete surface accommodation on the number flux, heat transfer coefficient, pressure coefficient, skin friction coefficient and total drag coefficient for a range of normal and tangential accommodation coefficients were investigated. The normal and tangential accommodation coefficients were varied from 1.0 to 0.5 , and the thickness of the frontal surface considered in this study covered hypersonic flow from the transitional flow regime to the free molecular flow regime.

Calculations showed that a reduction in the normal accommodation coefficient from 1.0 to 0.5 slightly increased the heat transfer coefficient at the vicinity of the stagnation point for the shapes investigated. In addition, it was found that a reduction in the tangential accommodation coefficient significantly diminished 

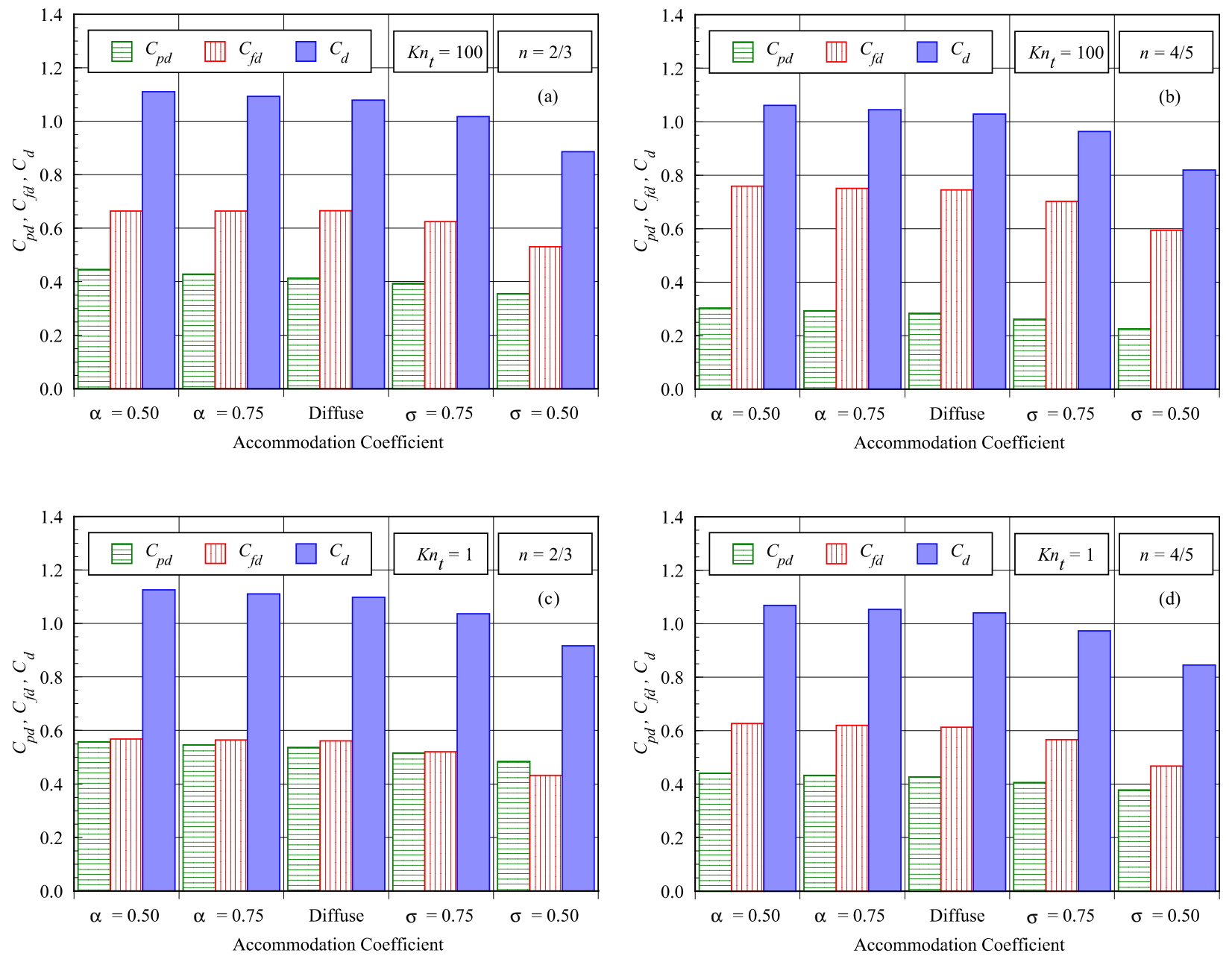

Figure 8. Effect of normal $\alpha_{n}$ and tangential $\sigma_{t}$ accommodation coefficients on pressure drag $C_{p d}$, skin friction $\operatorname{drag} C_{f d}$ and total drag coefficient $C_{f}$ along the body surface for (a) $K n_{t}=100$ and $n=2 / 3$, (b) $K n_{t}=100$ and $n=4 / 5$, (c) $K n_{t}=10$ and $n=2 / 3$, (d) $K n_{t}=10$ and $n=4 / 5$.

the heat transfer coefficient at the vicinity of the stagnation point for the leading edges investigated. Also, the analysis showed that the total drag coefficient is reduced by a reduction in the tangential accommodation coefficient, and slightly increased by a reduction in the normal accommodation coefficient. The effects of either normal or tangential accommodation coefficient showed that in order to make accurate predictions of the aerodynamic forces on, and heat transfer rates to, bodies in rarefied hypersonic flow it will be necessary to take surface accommodation into account. The calculations presented in this work have only covered a limited number of parametric variations. Further calculations with additional combinations of normal and tangential accommodation coefficients or where the internal energy accommodation is varied independently might provide more insight into the sensitivity of the aerodynamic surface quantities to gas-surface interaction model. 


\section{References}

${ }^{1}$ O'Brien, T. F. and Lewis, M. J., "Power Law Shapes for Leading-Edge Blunting with Minimal Shock Standoff," Journal of Spacecraft and Rockets, Vol. 36, No. 5, 1999, pp. 653-658.

${ }^{2}$ Santos, W. F. N., "Direct Simulation Monte Carlo of Rarefied Hypersonic Flow on Power Law Shaped Leading Edges," Ph.D. Dissertation, Dept. of Aerospace Engineering, University of Maryland, College Park, MD, Dec., 2001.

${ }^{3}$ Santos, W. F. N., and Lewis, M. J., "Power Law Shaped Leading Edges in Rarefied Hypersonic Flow," Journal of Spacecraft and Rockets, Vol. 39, No. 6, 2002, pp. 917-925.

${ }^{4}$ Santos, W. F. N., and Lewis, M. J., "Angle of Attack Effect on Rarefied Hypersonic Flow over Power Law Shaped Leading Edges," in 23rd International Symposium on Rarefied Gas Dynamics, Whistler, BC, Canada, July 20-25, 2002.

${ }^{5}$ Santos, W. F. N., and Lewis, M. J., "Shock Wave Structure in a Rarefied Hypersonic Flow on Power Law Shaped Leading Edges," in 41st AIAA Aerospace Sciences Meeting and Exhibit, AIAA Paper 2003-1134, Reno, NV, January 6-9, 2003.

${ }^{6}$ Santos, W. F. N., and Lewis, M. J., "Aerodynamic Heating Performance of Power Law Leading Edges in Rarefied Hypersonic Flow," in 36th AIAA Thermophysics Conference, AIAA Paper 2003-3894, Orlando, FL, June 23-26, 2003.

${ }^{7}$ Santos, W. F. N., and Lewis, M. J., "Effects of Compressibility on Rarefied Hypersonic Flow over Power Law Leading Edges," in 42nd AIAA Aerospace Sciences Meeting and Exhibit, AIAA Paper 2004-1181, Reno, NV, January 5-8, 2004.

${ }^{8}$ Santos, W. F. N., and Lewis, M. J., "DSMC Calculations of Rarefied Hypersonic Flow over Power Law Leading Edges with Incomplete Surface Accommodation," in 34th AIAA Fluid Dynamics Conference and Exhibit, AIAA Paper 2004-2636, Portland, OR, June 28-July 1, 2004.

${ }^{9}$ Santos, W. F. N., and Lewis, M. J., "Calculation of Shock Wave Structure over Power Law Bodies in Hypersonic Flow," Journal of Spacecraft and Rockets, Vol. 42, No. 2, 2005, pp. 213-222.

${ }^{10}$ Santos, W. F. N., and Lewis, M. J., "Aerothermodynamic Performance Analysis of Hypersonic Flow on Power Law Leading Edges," Journal of Spacecraft and Rockets, Vol. 42, No. 4, 2005, pp. 588-597.

${ }^{11}$ Boyd, I. D., and Padilla, J. F., "Simulation of Sharp Leading Edge Aerothermodynamic," in 12th AIAA International Space Planes and Hypersonic Systems and Technologies, AIAA Paper 2003-7062, Norfolk, VA, 15-19 December, 2003.

${ }^{12}$ Nonweiler, T. R. F., "Aerodynamic Problems of Manned Space Vehicles," Journal of the Royal Aeronautical Society, Vol. 63, Sept, 1959, pp. 521-528.

${ }^{13}$ Anderson, J. L., "Tethered Aerothermodynamic Research for Hypersonic Waveriders," in Proceedings of the 1st International Hypersonic Waverider Symposium, Univ. of Maryland, College Park, MD, 1990.

${ }^{14}$ Potter, J. L. and Rockaway, J. K., "Aerodynamic Optimization for Hypersonic Flight at Very High Altitudes," in Rarefied gas Dynamics: Space Science and Engineering, edited by B. D. Shizgal and D. P. Weaver, Vol. 160, Progress in Astronautics and Aeronautics, AIAA New York, 1994, pp.296-307.

${ }^{15}$ Rault, D. F. G., "Aerodynamic Characteristics of a Hypersonic Viscous Optimized Waverider at High Altitude," Journal of Spacecraft and Rockets, Vol. 31, No. 5, 1994, pp. 719-727.

${ }^{16}$ Graves, R. E. and Argrow, B. M., "Aerodynamic Performance of an Osculating-Cones Waverider at High Altitudes," in 35th AIAA Thermophysics Conference, AIAA Paper 2001-2960, Anaheim, CA, 2001.

${ }^{17}$ Shvets, A. I., Voronin, V. I., Blankson, I. M., Khikine, V. and Thomas, L., "On Waverider Performance with Hypersonic Flight Speed and High Altitudes," in 43rd AIAA Aerospace Sciences Meeting and Exhibit, AIAA Paper 2005-0512, Reno, NV, January 10-13, 2005.

${ }^{18}$ Mason, W. H. and Lee, J., "Aerodynamically Blunt and Sharp Bodies," Journal of Spacecraft and Rockets, Vol. 31, No. 3, 1994, pp. 378-382.

${ }^{19}$ Reller Jr., J. O., "Heat Transfer to Blunt Nose Shapes with Laminar Boundary Layers at High Supersonic Speeds," NACA RM-A57FO3a, 1957.

${ }^{20}$ Santos, W. F. N., "Aerothermodynamic Characteristics of Flat-Nose Power-Law Bodies in Low-Density Hypersonic Flow," in 22nd AIAA Applied Aerodynamics Conference and Exhibit, AIAA Paper 2004-5381, Providence, RI, 16-19 August, 2004.

${ }^{21}$ Santos, W. F. N., "Structure of Shock Wave on Flat-Nose Power-Law Bodies," in 43rd AIAA Aerospace Sciences Meeting and Exhibit, AIAA Paper 2005-0968, Reno, NV, January 10-13, 2005.

${ }^{22}$ Santos, W. F. N., "Some Extensions to the Aerothermodynamic Performance Study of Flat-Nose Power-Law Bodies," in 43rd AIAA Aerospace Sciences Meeting and Exhibit, AIAA Paper 2005-0969, Reno, NV, January 10-13, 2005.

${ }^{23}$ Lord, R. G., "Direct Simulation Monte Carlo Calculations of Rarefied Flows with Incomplete Surface Accommodation," in Journal of Fluid Mechanics, Vol. 239, 1992, pp. 449-459.

${ }^{24}$ Lord, R. G., "Application of the Cercignani-Lampis Scattering Kernel to Direct Simulation Monte Carlo Method," in 17th International Symposium on Rarefied Gas Dynamics, edited by A. E. Beylich, Aachen, Germany, pp. 1427-1433, July 8-14, 1991.

${ }^{25}$ Schaff, S. and Chambre P.,,Fundamentals of Gas Dynamics, Princeton University Press, Princeton, NJ, 1958.

${ }^{26}$ Cercignani, C. and Lampis, M., "Kinetic Models for Gas-Surface Interactions," Transport Theory and Statistical Physics, Vol. 1, No. 2, 1971, pp. 101-114.

${ }^{27}$ Bird, G. A., Molecular Gas Dynamics and the Direct Simulation of Gas Flows, Oxford University Press, Oxford, England, UK, 1994.

15 of 16 
${ }^{28}$ Bird, G. A., "Monte Carlo Simulation in an Engineering Context," in Progress in Astronautics and Aeronautics: Rarefied gas Dynamics, edited by Sam S. Fisher, Vol. 74, part I, AIAA New York, 1981, pp. 239-255.

${ }^{29}$ Bird, G. A., "Perception of Numerical Method in Rarefied Gasdynamics," in Rarefied gas Dynamics: Theoretical and Computational Techniques, edited by E. P. Muntz, and D. P. Weaver and D. H. Capbell, Vol. 118, Progress in Astronautics and Aeronautics, AIAA, New York, 1989, pp. 374-395.

${ }^{30}$ Borgnakke, C. and Larsen, P. S., "Statistical Collision Model for Monte Carlo Simulation of Polyatomic Gas Mixture," Journal of Computational Physics, Vol. 18, No. 4, 1975, pp. 405-420. 